\title{
An Enhanced Probabilistic Neural Network Approach Applied to Text Classification
}

\author{
Patrick Marques Ciarelli and Elias Oliveira \\ Universidade Federal do Espírito Santo \\ Av. Fernando Ferrari, 514 \\ 29075-910, Vitória - ES, Brazil \\ \{pciarelli, elias\}@lcad.inf.ufes.br
}

\begin{abstract}
Text classification is still a quite difficult problem to be dealt with both by the academia and by the industrial areas. On the top of that, the importance of aggregating a set of related amount of text documents is steadily growing in importance these days. The presence of multi-labeled texts and great quantity of classes turn this problem even more challenging. In this article we present an enhanced version of Probabilistic Neural Network using centroids to tackle the multi-label classification problem. We carried out some experiments comparing our proposed classifier against the other well known classifiers in the literature which were specially designed to treat this type of problem. By the achieved results, we observed that our novel approach were superior to the other classifiers and faster than the Probabilistic Neural Network without the use of centroids.
\end{abstract}

Keywords: Information Retrieval, Probabilistic Neural Network, Multi-label Problem.

\section{Introduction}

Automatic text classification is an activity that is becoming more and more important nowadays. This might be due to the huge amount of information available and the great challenge for the information retrieval. In addition, many of real databases are multilabeled and have a great amount of categories, which make the text classification task even more difficult [1]. Such problems are tackled by the information retrieval (IR) communities, both in academic and industrial contexts.

To treat such issues, in this paper we used a slightly modified version of the standard structure of the Probabilistic Neural Network (PNN) presented in [3]. In this modified version, we used centroids for the training of the PNN. In order to evaluate these PNN's versions, the classical one and that proposed by us in this paper, we used a set of multilabeled Yahoo's databases, initially used in [6]. Furthermore, we compared their results against the other specialized classifiers in multi-labeled classification. Both versions of the PNN achieved better results, in our evaluation, than that performed by the other classifiers. The enhanced PNN with centroids was the best in our evaluation.

This work is organized as follows. In Section 2 we detail our algorithms. We describe the metrics used to evaluate in Section 3 In Section 4 the experiments and results are discussed. Finally, we present our conclusions in Section 5 .

E. Bayro-Corrochano and J.-O. Eklundh (Eds.): CIARP 2009, LNCS 5856, pp. 661-668, 2009.

(c) Springer-Verlag Berlin Heidelberg 2009 


\section{The Algorithms}

The Probabilistic Neural Network is an artificial neural network for nonlinear computing which approaches the Bayes optimal decision boundaries. The original PNN algorithm [2] was designed for single-label problems. Thus, its standard architecture was slightly modified, so that it is now capable of solving multi-labeled problems.

In this modified version, instead of four, the PNN is composed of only three layers: the input layer, the pattern layer and the summation layer, as it is showed in Figure 1 Thus, like in the original structure, this version of PNN needs only one training step, therefore, its training is faster than other well known feed-forward neural networks [4].

The training consists in assigning each training sample $w_{j}$ of category $C_{j}$ to a neuron of pattern layer of category $C_{j}$. Thus, the weight vector of this neuron is the characteristics vector of the sample.

For each $d_{j}$ test instance passed by the input layer to a neuron in the pattern layer, it computes the output for the $d_{j}$. The computation is showed in Equation[1.

$$
F_{k, i}\left(d_{j}\right)=\frac{1}{2 \pi \sigma^{2}} \exp \left(\frac{d_{j}^{t} w_{k, i}-1}{\sigma^{2}}\right),
$$

where the $d_{j}$ is the pattern characteristics input vector, and the $w_{k, i}$ is the $k^{\text {th }}$ sample for a neuron of category $C_{i}, k \in N_{i}$, whereas $N_{i}$ is the number of neurons of $C_{i}$. In addition, $d_{j}$ and $w_{k, i}$ were normalized so that $d_{j}^{t} d_{j}=1$ and $w_{k, i}^{t} w_{k, i}=1$. The $\sigma$ is the Gaussian standard deviation, which determines the receptive field of the Gaussian curve.

The next step is the summation layer. In this layer, all outputs of the previous layer are summed, Equation 2 in each cluster $C_{i}$ producing $p_{i}\left(d_{j}\right)$ values, where $|C|$ is the total number of categories and $h_{i}$ is the priori probability of the class $C_{i}$. Whether we consider the priori probability from database of training, so we can inconsiderate the fraction $\frac{h_{i}}{N_{i}}$.

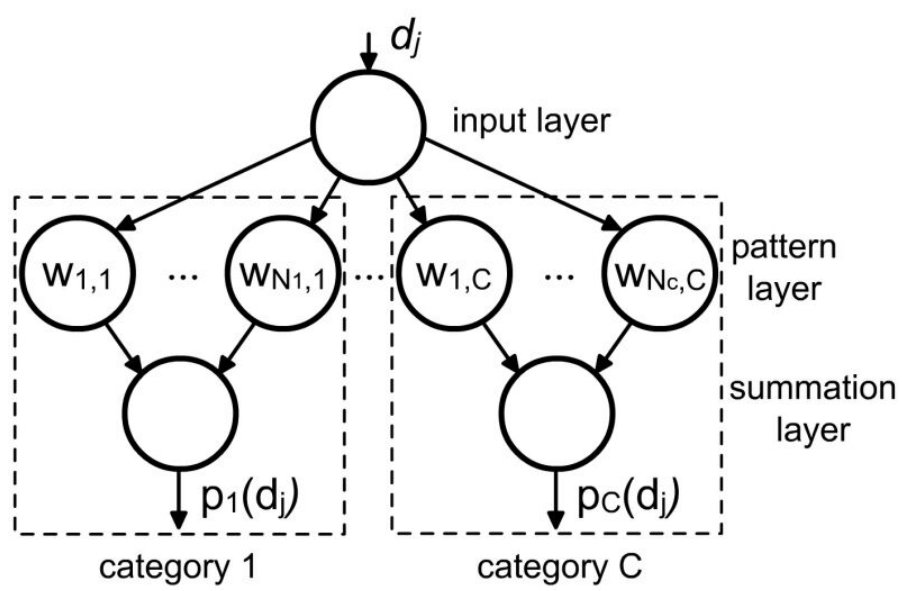

Fig. 1. The modified Probabilistic Neural Network architecture 


$$
p_{i}\left(d_{j}\right)=\frac{h_{i}}{N_{i}} \sum_{k=1}^{N_{i}} F_{k, i}\left(d_{j}\right) \quad i=1,2, \ldots,|C| .
$$

Finally, for the selection of the categories which will be assigned by the neural network to each sample, we consider the most likely categories pointed out by the summation layer based on a chosen threshold.

Differently from other types of networks, such as those feed forward based, the proposed PNN needs few parameters to be configured: the $\sigma$, (see in Equation 1) and the determination of threshold value. Another advantage of the probabilistic neural networks is that it is easy to add new categories, or new training inputs, into the already running structure, which is good for the on-line applications [4]. On the other hand, one of its drawbacks is the great number of neurons in the pattern layer that, as a consequence, may produce a high consumption of memory and slow rate of classification. Moreover, the presence of repeated samples may harm the performance of the classifier.

\subsection{Probabilistic Neural Network with Centroids}

To minimize the drawbacks of the PNN, we propose a technique of centroids, such that is used only one neuron for class in the pattern layer. Equation 3 shows a mathematical procedure to obtain the centroid for each class $C_{i}$, where $w_{k, i}$ is the $k^{\text {th }}$ sample of training of class $C_{i}, N_{i}$ is the number of samples of $C_{i}$ and $W_{i}$ is the obtained centroid. To reduce the loss of information, we also obtain from database the priori probability $h_{i}$ of each class. Thus, the fraction $\frac{h_{i}}{N_{i}}$ of Equation 2 will be reduced to $h_{i}$, because $N_{i}$ will be equal to 1 , and the centroid $W_{i}$ is associated to the neuron of pattern layer of class $C_{i}$. Hence, with this procedure, the PNN will have only one neuron per category in the pattern layer.

$$
W_{i}=\frac{1}{N_{i}} \sum_{k=1}^{N_{i}} w_{k, i} \quad h_{i}=N_{i} \quad i=1, \ldots,|C| .
$$

\section{Metrics}

Formalizing the problem we have at hand, text categorization may be defined as the task of assigning documents to a predefined set of categories, or classes [1]. In multilabel text categorization a document may be assigned to one or more categories. Let $\mathcal{D}$ be the domain of documents, $\mathcal{C}=\left\{c_{1}, c_{2}, \ldots, c_{|C|}\right\}$ a set of pre-defined categories, and $\Omega=\left\{d_{1}, d_{2}, \ldots, d_{|\Omega|}\right\}$ an initial corpus of documents previously categorized by some human specialists into subsets of categories of $C$.

In multi-label learning, the training(-and-validation) set $T V=\left\{d_{1}, d_{2}, \ldots, d_{|T V|}\right\}$ is composed of a number documents, each associated with a subset of categories in $C . T V$ is used to train and validate (actually, to tune eventual parameters of) a categorization system that associates the appropriate combination of categories to the characteristics of each document in the $T V$. The test set $T e=\left\{d_{|T V|+1}, d_{|T V|+2}, \ldots, d_{|\Omega|}\right\}$, on the other hand, consists of documents for which the categories are unknown to the automatic categorization systems. $T V$ has $|T V|$ samples and $T e$ has $|\Omega|-|T V|=p$ samples. After 
being trained, as well as tuned, by the $T V$, the categorization systems are used to predict the set of categories of each document in $T e$.

A multi-label categorization system typically implements a real-valued function of the form $f: \mathcal{D} \times \mathcal{C} \rightarrow \mathbb{R}$ that returns a value for each pair $\left\langle d_{j}, c_{j}\right\rangle \in \mathcal{D} \times \mathcal{C}$ that, roughly speaking, represents the evidence for the fact that the test document $d_{j}$ should be categorized under the category $c_{j} \in C_{j}$, where $C_{j} \subset \mathcal{C}$. The real-valued function $f(.,$. can be transformed into a ranking function $r(.,$.$) , which is an one-to-one mapping onto$ $\{1,2, \ldots,|C|\}$ such that, if $f\left(d_{j}, c_{1}\right)>f\left(d_{j}, c_{2}\right)$, then $r\left(d_{j}, c_{1}\right)<r\left(d_{j}, c_{2}\right)$. If $C_{j}$ is the set of proper categories for the test document $d_{j}$, then a successful categorization system tends to rank categories in $C_{j}$ higher than those not in $C_{j}$. Additionally, we also use a threshold parameter so that those categories that are ranked above the threshold $\tau$ (i.e., $\left.c_{k} \mid f\left(d_{j}, c_{k}\right) \geq \tau\right)$ are the only ones to be assigned to the test document.

We have used five multi-label metrics discussed in [6] to evaluate the performance of classifiers. We now present each one of these metrics:

Hamming Loss (hloss) evaluates how many times the test document $d_{j}$ is misclassified, i.e., a category not belonging to the document is predicted or a category belonging to the document is not predicted.

$$
\text { hloss }=\frac{1}{p} \sum_{j=1}^{p} \frac{1}{|C|}\left|P_{j} \Delta C_{j}\right|
$$

where $|C|$ is the number of categories and $\Delta$ is the symmetric difference between the set of predicted categories $P_{j}$ and the set of appropriate categories $C_{j}$ of the test document $d_{j}$. The predicted categories are those which rank higher than the threshold $\tau$.

One-error (one-error) evaluates if the top ranked category is present in the set of appropriate categories $C_{j}$ of the test document $d_{j}$.

$$
\text { one-error }=\frac{1}{p} \sum_{j=1}^{p} \text { error }_{j}, \quad \text { error }_{j}=\left\{\begin{array}{l}
0 \text { if }\left[\arg \max _{c \in C} f\left(d_{j}, c\right)\right] \in C_{j} \\
1 \text { otherwise. }
\end{array}\right.
$$

where [arg $\left.\max _{c \in C} f\left(d_{j}, c\right)\right]$ returns the top ranked category for the test document $d_{j}$.

Coverage (coverage) measures how far we need to go down the rank of categories in order to cover all the possible categories assigned to a test document.

$$
\text { coverage }=\frac{1}{p} \sum_{j=1}^{p}\left(\max _{c \in C_{j}} r\left(d_{j}, c\right)-1\right),
$$

where $\max _{c \in C_{j}} r\left(d_{j}, c\right)$ returns the maximum rank for the set of appropriate categories of the test document $d_{j}$.

Ranking Loss (rloss) evaluates the fraction of category pairs $\left\langle c_{k}, c_{l}\right\rangle$, for which $c_{k} \in$ $C_{j}$ and $c_{l} \in \bar{C}_{j}$, that are reversely ordered for the test document $d_{j}$ :

$$
\text { rloss }=\frac{1}{p} \sum_{j=1}^{p} \frac{\left|\left\{\left(c_{k}, c_{l}\right) \mid f\left(d_{j}, c_{k}\right) \leq f\left(d_{j}, c_{l}\right)\right\}\right|}{\left|C_{j}\right|\left|\bar{C}_{j}\right|}
$$

where $\left(c_{k}, c_{l}\right) \in C_{j} \times \bar{C}_{j}$, and $\bar{C}_{j}$ is the complementary set of $C_{j}$ in $C$. 
Average Precision (avgprec) evaluates the average of precision computed after truncating the ranking of categories after each category $c_{i} \in C_{j}$ in turn:

$$
\operatorname{avgprec}=\frac{1}{p} \sum_{j=1}^{p} \frac{1}{\left|C_{j}\right|} \sum_{k=1}^{\left|C_{j}\right|} \operatorname{precision}_{j}\left(R_{j k}\right),
$$

where $R_{j k}$ is the set of ranked categories that goes from the top ranked category until a ranking position $k$, where there is a category $c_{i} \in C_{j}$ for $d_{j}$, and $\operatorname{precision}_{j}\left(R_{j k}\right)$ is the number of pertinent categories in $R_{j k}$ divided by $\left|R_{j k}\right|$.

The smaller the value of hamming loss, one-error, coverage and ranking loss, and the larger the value of average precision, the better the performance of the categorization system. The performance is optimal when hloss $=$ one-error $=$ rloss $=0$ and avgprec $=1$.

\section{Experiments}

We carry out a series of experiments to compare the versions of PNN against the classifiers: ML-kNN, that is based on the kNN [6], Rank-SVM [9], a modified version of SVM, ADTBoost.MH [7] and BoosTexter [8], that both are techniques based on decision trees. We have used 11 text databases from Yahoo domain in our experiment 1 , where each database has 2000 samples to training and 3000 to test, the average number of classes is 30 and there is a mean of 1.48 classes assigned by sample. To evaluate

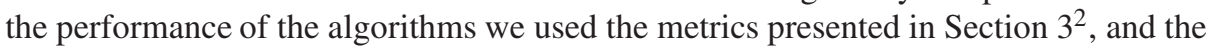
results were obtained directly from the [6], with exception of the PNNs' results.

In [6] is not mentioned any use of a search strategy for the optimization of the classifiers' parameters. To turn it in a fair comparison with the other techniques, we will test our approaches of PNNs considering only the order of magnitude of the variance's value. For this, we used part of training set of Arts database from Yahoo and we tested the variance's values 10,1 and 0.1 on a cross-validation experiment. The chosen value was 0.1. The threshold's value used to the Hamming Loss metric was 0.5, the same value used by ML-kNN, therefore, this parameter was also not optimized to the PNNs.

The results yielded with the use of the Yahoo's database are presented in Tables from 1 to 5 Each one of the tables represents a metric, where each row is a data set and each column is a classifier. The term "Average" in the last row means the average value of the metric obtained by each classifier to all databases.

To accomplish a clearer evaluation of the classifiers, we adopted two criteria derived from [6]. The first criterion creates one partial order " $\succ$ " that evaluates the performance between two classifiers for each metric. In that way, if the classifier $A 1$ has a better performance than $A 2$ to a given metric, so we have $A 1 \succ A 2$. In order to perform this task, we used two-tailed paired t-test at $5 \%$ significance level.

However, the presented criterion is insufficient to obtain the performance of classifiers as a whole, therefore, we used a second criterion. In this one is applied a system

\footnotetext{
${ }^{1}$ Databases and codes of the versions of PNN are available at http://www.inf.ufes.br/ elias/ciarp2009.zip

2 Ranking Loss to ADTBoost.MH was not reported because, according to [6], the algorithm of this classifier did not supply such information.
} 
Table 1. Hamming Loss obtained by the classifiers

\begin{tabular}{|c|c|c|c|c|c|c|}
\hline Data Set & $\overline{\mathrm{ML}-\mathrm{kNN}}$ & BoosTexter & ADTBoost.MH & Rank-SVM & $\overline{\mathrm{PNN}}$ & PNN-centroid \\
\hline Arts\&Humanities & 0.0612 & 0.0652 & 0.0585 & 0.0615 & 0.0630 & 0.0626 \\
\hline Business\&Economy & 0.0269 & 0.0293 & 0.0279 & 0.0275 & 0.0307 & 0.0289 \\
\hline Computers\&Internet & 0.0412 & 0.0408 & 0.0396 & 0.0392 & 0.0447 & 0.0412 \\
\hline Education & 0.0387 & 0.0457 & 0.0423 & 0.0398 & 0.0437 & 0.0437 \\
\hline Entertainment & 0.0604 & 0.0626 & 0.0578 & 0.0630 & 0.0640 & 0.0635 \\
\hline Health & 0.0458 & 0.0397 & 0.0397 & 0.0423 & 0.0514 & 0.0481 \\
\hline Recreation\&Sports & 0.0620 & 0.0657 & 0.0584 & 0.0605 & 0.0634 & 0.0631 \\
\hline Reference & 0.0314 & 0.0304 & 0.0293 & 0.0300 & 0.0307 & 0.0289 \\
\hline Science & 0.0325 & 0.0379 & 0.0344 & 0.0340 & 0.0353 & 0.0353 \\
\hline Social\&Science & 0.0218 & 0.0243 & 0.0234 & 0.0242 & 0.0281 & 0.0245 \\
\hline Society\&Culture & 0.0537 & 0.0628 & 0.0575 & 0.0555 & 0.0596 & 0.0599 \\
\hline Average & 0.0432 & 0.0459 & 0.0426 & 0.0434 & 0.0468 & 0.0454 \\
\hline
\end{tabular}

Table 2. One-Error obtained by the classifiers

\begin{tabular}{ccccccc}
\hline Data Set & \multicolumn{1}{c}{ ML-kNN } & BoosTexter & ADTBoost.MH & Rank-SVM & PNN & PNN-centroid \\
\hline Arts\&Humanities & 0.6330 & 0.5550 & 0.5617 & 0.6653 & 0.5597 & $\mathbf{0 . 5 2 9 3}$ \\
Business\&Economy & $\mathbf{0 . 1 2 1 3}$ & 0.1307 & 0.1337 & 0.1237 & 0.1317 & 0.1313 \\
Computers\&Internet & 0.4357 & 0.4287 & 0.4613 & $\mathbf{0 . 4 0 3 7}$ & 0.4457 & 0.4557 \\
Education & 0.5207 & 0.5587 & 0.5753 & $\mathbf{0 . 4 9 3 7}$ & 0.5463 & 0.5420 \\
Entertainment & 0.5300 & $\mathbf{0 . 4 7 5 0}$ & 0.4940 & 0.4933 & 0.5530 & 0.4960 \\
Health & 0.4190 & $\mathbf{0 . 3 2 1 0}$ & 0.3470 & 0.3323 & 0.4080 & 0.3807 \\
Recreation\&Sports & 0.7057 & 0.5557 & $\mathbf{0 . 5 5 4 7}$ & 0.5627 & 0.6037 & 0.5670 \\
Reference & 0.4730 & 0.4427 & 0.4840 & $\mathbf{0 . 4 3 2 3}$ & 0.4780 & 0.4727 \\
Science & 0.5810 & 0.6100 & 0.6170 & $\mathbf{0 . 5 5 2 3}$ & 0.6123 & 0.5930 \\
Social\&Science & $\mathbf{0 . 3 2 7 0}$ & 0.3437 & 0.3600 & 0.3550 & 0.3753 & 0.3703 \\
Society\&Culture & 0.4357 & 0.4877 & 0.4845 & $\mathbf{0 . 4 2 7 0}$ & 0.4647 & 0.4637 \\
\hline Average & 0.4711 & 0.4463 & 0.4612 & $\mathbf{0 . 4 4 0 1}$ & 0.4708 & 0.4547 \\
\hline
\end{tabular}

Table 3. Coverage obtained by the classifiers

\begin{tabular}{ccccccc}
\hline Data Set & ML-kNN & BoosTexter & ADTBoost.MH & Rank-SVM & PNN & PNN-centroid \\
\hline Arts\&Humanities & 5.4313 & 5.2973 & 5.1900 & 9.2723 & 4.8503 & $\mathbf{4 . 6 2 5 0}$ \\
Business\&Economy & 2.1840 & 2.4123 & 2.4730 & 3.3637 & 2.1087 & $\mathbf{2 . 0 5 2 7}$ \\
Computers\&Internet & 4.4117 & 4.4887 & 4.4747 & 8.7910 & 4.0380 & $\mathbf{3 . 8 9 6 3}$ \\
Education & 3.4973 & 4.0673 & 3.9663 & 8.9560 & 3.4980 & $\mathbf{3 . 4 0 6 7}$ \\
Entertainment & 3.1467 & 3.0883 & 3.0877 & 6.5210 & 3.0663 & $\mathbf{2 . 8 8 8 3}$ \\
Health & 3.3043 & 3.0780 & 3.0843 & 5.5400 & 3.0093 & $\mathbf{2 . 8 7 3 0}$ \\
Recreation\&Sports & 5.1010 & 4.4737 & 4.3380 & 5.6680 & 4.2773 & $\mathbf{4 . 0 5 7 3}$ \\
Reference & 3.5420 & 3.2100 & 3.2643 & 6.9683 & 2.9097 & $\mathbf{2 . 7 5 6 0}$ \\
Science & 6.0470 & 6.6907 & 6.6027 & 12.401 & 5.9930 & $\mathbf{5 . 6 1 8 0}$ \\
Social\&Science & 3.0340 & 3.6870 & 3.4820 & 8.2177 & 3.1357 & $\mathbf{2 . 9 4 3 0}$ \\
Society\&Culture & 5.3653 & 5.8463 & $\mathbf{4 . 9 5 4 5}$ & 6.8837 & 5.3350 & 5.2323 \\
\hline Average & 4.0968 & 4.2127 & 4.0834 & 7.5075 & 3.8383 & $\mathbf{3 . 6 6 8 1}$ \\
\hline
\end{tabular}

Table 4. Ranking Loss obtained by the classifiers

\begin{tabular}{ccccccc}
\hline Data Set & ML-kNN & BoosTexter & ADTBoost.MH & Rank-SVM & PNN & PNN-centroid \\
\hline Arts\&Humanities & 0.1514 & 0.1458 & N/A & 0.2826 & 0.1306 & $\mathbf{0 . 1 2 2 3}$ \\
Business\&Economy & 0.0373 & 0.0416 & N/A & 0.0662 & 0.0367 & $\mathbf{0 . 0 3 4 9}$ \\
Computers\&Internet & 0.0921 & 0.0950 & N/A & 0.2091 & 0.0826 & $\mathbf{0 . 0 7 8 7}$ \\
Education & 0.0800 & 0.0938 & N/A & 0.2080 & 0.0803 & $\mathbf{0 . 0 7 7 3}$ \\
Entertainment & 0.1151 & 0.1132 & N/A & 0.2617 & 0.1103 & $\mathbf{0 . 1 0 2 5}$ \\
Health & 0.0605 & 0.0521 & N/A & 0.1096 & 0.0526 & $\mathbf{0 . 0 4 9 1}$ \\
Recreation\&Sports & 0.1913 & 0.1599 & N/A & 0.2094 & 0.1556 & $\mathbf{0 . 1 4 3 2}$ \\
Reference & 0.0919 & 0.0811 & N/A & 0.1818 & 0.0732 & $\mathbf{0 . 0 6 8 5}$ \\
Science & 0.1167 & 0.1312 & N/A & 0.2570 & 0.1166 & $\mathbf{0 . 1 0 7 3}$ \\
Social\&Science & 0.0561 & 0.0684 & N/A & 0.1661 & 0.0601 & $\mathbf{0 . 0 5 4 6}$ \\
Society\&Culture & 0.1338 & 0.1483 & N/A & 0.1716 & 0.1315 & $\mathbf{0 . 1 2 8 6}$ \\
\hline Average & 0.1024 & 0.1028 & N/A & 0.1930 & 0.0936 & $\mathbf{0 . 0 8 7 9}$ \\
\hline
\end{tabular}


Table 5. Average Precision obtained by the classifiers

\begin{tabular}{ccccccc}
\hline Data Set & ML-kNN & BoosTexter & ADTBoost.MH & Rank-SVM & PNN & PNN-centroid \\
\hline Arts\&Humanities & 0.5097 & 0.5448 & 0.5526 & 0.4170 & 0.5645 & $\mathbf{0 . 5 8 5 1}$ \\
Business\&Economy & $\mathbf{0 . 8 7 9 8}$ & 0.8697 & 0.8702 & 0.8694 & 0.8763 & 0.8779 \\
Computers\&Internet & 0.6338 & $\mathbf{0 . 6 4 4 9}$ & 0.6235 & 0.6123 & 0.6398 & 0.6420 \\
Education & $\mathbf{0 . 5 9 9 3}$ & 0.5654 & 0.5619 & 0.5702 & 0.5889 & 0.5980 \\
Entertainment & 0.6013 & $\mathbf{0 . 6 3 6 8}$ & 0.6221 & 0.5637 & 0.5991 & 0.6295 \\
Health & 0.6817 & $\mathbf{0 . 7 4 0 8}$ & 0.7257 & 0.6839 & 0.7047 & 0.7207 \\
Recreation\&Sports & 0.4552 & 0.5572 & 0.5639 & 0.5315 & 0.5396 & $\mathbf{0 . 5 6 7 2}$ \\
Reference & 0.6194 & $\mathbf{0 . 6 5 7 8}$ & 0.6264 & 0.6176 & 0.6441 & 0.6512 \\
Science & $\mathbf{0 . 5 3 2 4}$ & 0.5006 & 0.4940 & 0.5007 & 0.5073 & 0.5278 \\
Social\&Science & $\mathbf{0 . 7 4 8 1}$ & 0.7262 & 0.7217 & 0.6788 & 0.7113 & 0.7272 \\
Society\&Culture & $\mathbf{0 . 6 1 2 8}$ & 0.5717 & 0.5881 & 0.5717 & 0.5993 & 0.6018 \\
\hline Average & 0.6249 & 0.6378 & 0.6318 & 0.6015 & 0.6341 & $\mathbf{0 . 6 4 8 0}$ \\
\hline & & & & &
\end{tabular}

Table 6. Relative performance of the classifiers by the two criteria

\begin{tabular}{|c|c|}
\hline $\begin{array}{l}\text { HL-Hamming L } \\
\text { A1-ML-kNN; A }\end{array}$ & $\begin{array}{l}\text { oss; OE-One-error; C-Coverage; RL-Ranking Loss; AP-Average Precision } \\
\text { 2-BoosTexter; A3-ADTBoost.MH; A4-Rank-SVM; A5-PNN; A6-PNN-centroid }\end{array}$ \\
\hline Metrics & Criterion 1 \\
\hline HL & $\overline{A 1} \succ A 5, A 1 \succ A 6, A 3 \succ A 2, A 4 \succ A 2, A 3 \succ A 5, A 3 \succ A 6, A 4 \succ A 5, A 4 \succ A 6, A 6 \succ A 5$ \\
\hline $\mathrm{OE}$ & $A 2 \succ A 3, A 2 \succ A 5, A 6 \succ A 5$ \\
\hline $\mathrm{C}$ & $\begin{array}{l}A 1 \succ A 4, A 5 \succ A 1, A 6 \succ A 1, A 2 \succ A 4, A 5 \succ A 2, A 6 \succ A 2, A 3 \succ A 4, A 5 \succ A 3, A 6 \succ A 3, \\
A 5 \succ A 4, A 6 \succ A 4, A 6 \succ A 5\end{array}$ \\
\hline RL & $A 1 \succ A 4, A 5 \succ A 1, A 6 \succ A 1, A 2 \succ A 4, A 5 \succ A 2, A 6 \succ A 2, A 5 \succ A 4, A 6 \succ A 4, A 6 \succ A 5$ \\
\hline $\mathrm{AP}$ & $A 2 \succ A 4, A 3 \succ A 4, A 6 \succ A 3, A 5 \succ A 4, A 6 \succ A 4, A 6 \succ A 5$ \\
\hline Techniques & $\begin{array}{c}\text { Criterion 2 } \\
\end{array}$ \\
\hline ML-kNN & $\{$ PNN, PNN-centroid $\}>$ ML-kNN > Rank-SVM \\
\hline BoosTexter & $\{$ PNN,PNN-centroid $\}>$ BoosTexter $>$ Rank-SVM \\
\hline ADTBoost.MH & PNN-centroid > ADTBoost.MH > Rank-SVM \\
\hline Rank-SVM & $\{$ ML-kNN, BoosTexter, ADTBoost.MH,PNN, PNN-centroid $\}>$ Rank-SVM \\
\hline PNN & PNN-centroid $>$ PNN $>\{$ ML-kNN, BoosTexter, Rank-SVM $\}$ \\
\hline PNN-centroid & PNN-centroid $>$ \{ML-kNN, BoosTexter, Rank-SVM, ADTBoost.MH, PNN $\}$ \\
\hline
\end{tabular}

based on rewards and punishes. For example, for the case of $A 1 \succ A 2$ the classifier $A 1$ is rewarded with +1 and the classifier $A 2$ is punished with -1 . Then, we compare the classifiers two a two through of the sum of their rewarded and punished between them. In this case, if $A 1$ have a positive value in relation to $A 2$, so $A 1$ is superior to $A 2, i$. $e$, $A 1>A 2$. Thus, the results obtained by the two criteria are shown in Table 6 .

Table 6] shows that PNN-centroid is superior over to every algorithm, while the RankSVM is the worst. Moreover, the PNN shows to be the second better, being inferior just to the PNN-centroid and it had similar performance to ADTBoost.MH, whereas the other classifiers (ML-kNN, ADTBoost.MH and BoosTexter) were superior just to Rank-SVM. In addition, the PNN-centroid had a low time for classification (more than 10 times faster) and a small consumption of memory, when we compared with the PNN. Finally, the training phase of both PNNs was faster than the other algorithms.

\section{Conclusions}

The problem of text classification is still greatly challenging, due to the huge amount of information available. Other issues are the great quantity of classes and the presence of multi-labeled databases, which together increase the difficult of this task.

In this work, we presented an experimental evaluation on multi-label text classification of the performance of Probabilistic Neural Network and another version of it 
with centroids. Therefore, we conducted a comparative study of these PNNs and other four classifiers specially designed to solve this problem. The results showed that both versions of PNN devised by us presented good results, specially the PNN with centroids, that was superior to all the other classifiers. In addition, our approach is faster and consumed less memory than PNN without centroids.

A direction for future works is to study methods that can improve the results found in this article even more. Moreover, we are planning to tackle the problem of on-line learning using the proposed neural network in this paper.

\section{Aknowledgement}

This work was parcially supported by Receita Federal do Brasil and Fundação Espírito Santense de Tecnologia - FAPES-Brasil (grant 41936450/2008).

\section{References}

1. Sebastiani, F.: Machine learning in automated text categorization. ACM Computing Surveys 34(1), 1-47 (2002)

2. Specht, D.: Probabilistic Neural Networks, Oxford, UK, vol. 3(1), pp. 109-118. Elsevier Science Ltd., Amsterdam (1990)

3. Oliveira, E., Ciarelli, P.M., Badue, C., De Souza, A.F.: A Comparison between a KNN Based Approach and a PNN Algorithm for a Multi-label Classification Problem. In: ISDA 2008: Eighth International Conference on Intelligent Systems Design and Applications, vol. 2, pp. 628-633 (2008)

4. Duda, R.O., Hart, P.E., Stork, D.G.: Pattern Classification, 2nd edn. Wiley-Interscience, New York (2001)

5. Baeza-Yates, R., Ribeiro-Neto, B.: Modern Information Retrieval, 1st edn. Addison-Wesley, New York (1998)

6. Zhang, M.-L., Zhou, Z.-H.: MLkNN: A Lazy Learning Approach to Multi-Label Learning. Pattern Recognition 40(1), 2038-2048 (2007)

7. De Comité, F., Gilleron, R., Tommasi, M.: Learning Multi-label Alternating Decision Trees from Texts and Data. LNCS, pp. 35-49. Springer, Heidelberg (2003)

8. Schapire, R.E., Singer, Y.: BoosTexter: A Boosting-based System for Text Categorization. Machine Learning, 135-168 (2000)

9. Elisseeff, A., Weston, J.: Kernel Methods for Multi-labelled Classification and Categorical Regression Problems. In: Advances in Neural Information Processing Systems, pp. 681-687 (2001) 